Received: July 25, 2017

Revision received: June 2, 2018

\title{
Evaluation of Computer Assisted Translation and its Teaching Application in English Translation
}

\author{
Xiaoyan $\mathrm{Wu}^{1}$ \\ Yan'an University
}

\author{
Shuili Zhang ${ }^{2}$ \\ Yan'an University
}

\begin{abstract}
This paper first presents the definition of computer assisted translation (CAT), usability index, user experience. Combining quantitative analysis method and questionnaire investigation method, this paper evaluates the application of CAT based on usability index and user experience. Research hypothesis, research design, model architecture and hypothesis validation model are used to verify the impact of usability index on users' choice of CAT software. Finally, the paper investigates the application of CAT software in English translation teaching for college students in China, and collects and analyzes the attitudes of teachers and students to CAT. The paper studies the contradiction between user demand and CAT software supply from the perspective of the usability of CAT so that to help the user to choose appropriate CAT software. The research on the application of CAT in English teaching has a scientific effect on the promotion of CAT in English translation.
\end{abstract}

\section{Keywords}

CAT Software • Computer Assisted Translation (CAT) • Machine Translation • Usability Index • User Experience

\footnotetext{
${ }^{1}$ Correspondence to: Xiaoyan Wu. School of Foreign Languages, Yan'an University, Yan'an 716000, China. Email: yadxxiaoyan@126.com

${ }^{2}$ School of Physics and Electronic Information, Yan'an University, Yan'an 716000, China. Email: zhangshuili74@163.com

\begin{tabular}{l} 
Citation: Wu, X. Y., Zhang, S. L. (2018). Evaluation of Computer Assisted Translation and its Teaching Application in \\
English Translation. Educational $\quad$ Sciences: Theory \& Practice, 18(5), \\
http://dx.doi.org/10.12738/estp.2018.5.099 \\
\hline
\end{tabular}


With the acceleration and deepening of internationalization, the communication demands among people of different languages in the world are becoming more and more intense, while the translation industry, as the bridge of communication among countries, has gained tremendous development this year. Stimulated by the rapid development of market and computer technology, CAT has replaced the traditional machine translation to become a main translation tool in translation industry in order to meet the diverse, individualized and mass demand. The work mode of translation industry has also developed into a team practice mode, and the mastery of CAT software has become the basic quality of translators. In order to cultivate mature translation talents, majors related to CAT have been set up at the university education stage (Hutchins, \& Somers, 1992; Zhang \& Zhang, 2013; Barrachina et al., 2009).

At present, both the domestic and foreign markets are flooded with a variety of CAT software. Besides Trados under SDL company, Déjà Vu under Atiral company, Snowman CAT software and other mainstream translation software, there are still other types of CAT software, which may confuse translators in terms of economy and practicability when choosing and using the translation software. The present researches on CAT mainly focus on the one-way and technical research of the software itself, while few researches can be

found on the usability evaluation of CAT software. This paper first presents the definition of computer assisted translation (CAT), usability index, user experience. Combining quantitative analysis method and questionnaire investigation method, this paper evaluates the application of CAT based on usability indexes and user experience. Hypothesis validation model is used to verify the impact of usability indexes, including satisfaction, error rate, learning cycle, efficiency on users' choice of CAT software. Finally, the paper analyzes the application of CAT software in English translation teaching through researches and investigations on the students majored in CAT translation in universities. This paper makes a scientific guidance for users to select more appropriate CAT software, and has a positive role in promoting the application of CAT in English translation teaching (Esplà-Gomis, Sánchez-Martínez \& Forcada, 2015; Mileto, 2014; Vakil, \& Khadivi, 2013).

\section{Concept Definition}

\section{Computer assisted translation}

Computer assisted translation (CAT) is different from machine translation. Machine translation aims to completely replace people to undertake the translation work, while CAT aims at using a series of translation tools to assist the translator to complete the work. Unlike machine translation, the outcome of CAT will not be completely transformed into an unrecognized one. Its main purpose is to serve as a translation aid in order to reduce the labour intensity of the translator by eliminating unnecessary repetitions. CAT is composed of translation memory and terminology database. Translation memory records the results according to human translation. Once meeting a similar translation text similar, the system can stimulate the translation memory so that to assist translation. Termbase is similar to the translation memory, which refers to standard translation samples established by the translators themselves.

The idea of computer translation is derived from the $1970 \mathrm{~s}$, the idea of which is to take advantage of the 
powerful retrieval and storage functions of the computer to avoid unnecessary repeated translation. The continuing rising demand of computer assisted translation software in the market brings stimulation to software developers. New CAT software is launched into market every year, among which Trados under SDL company, Déjà Vu of Atiral, Yaxin CAT, and Snowman CAT software are widely used. Users continue to demand more for the economy, functionality and usability of the software.

Figure 1 is a screenshot of the Chinese interface of Trados. Users can set up translation projects for different translation tasks and improve the accuracy and efficiency of translation with the help of Trados.

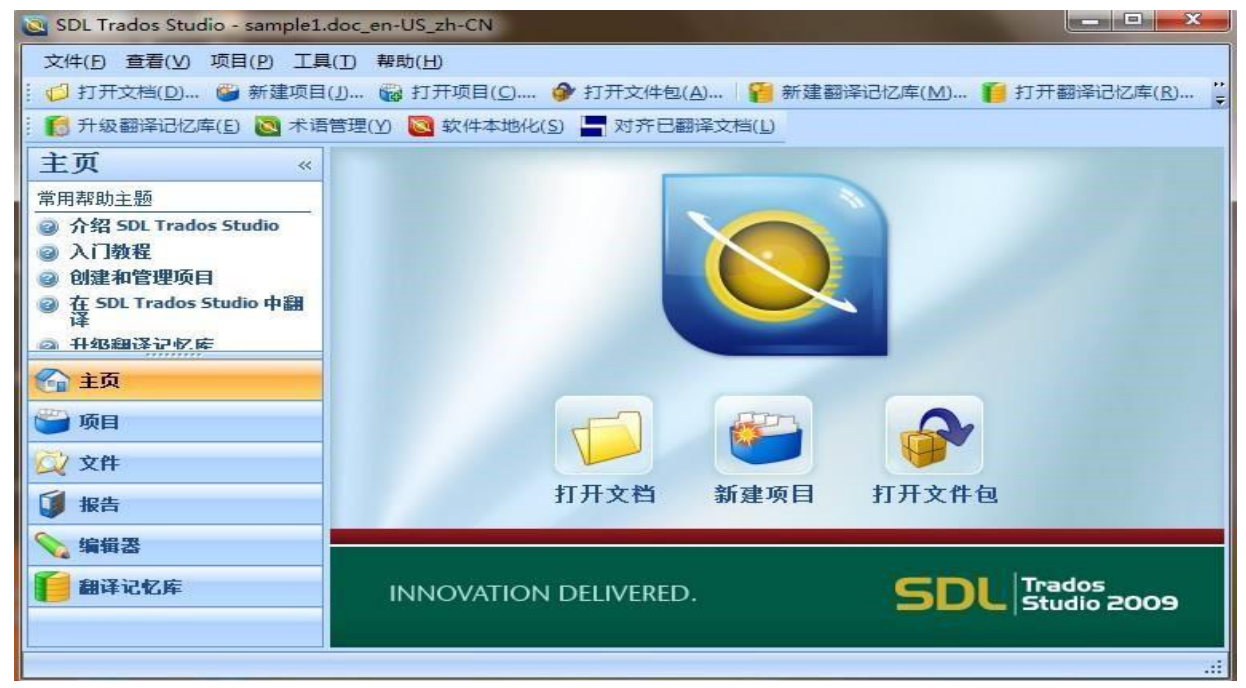

Figure 1. The interface of CAT software "Trados"

\section{Usability indexes}

The usability of computer assisted translation software is mainly embodied in the following five aspects:

(1) Learnability: learners can learn the operation of translation software in a relatively short period of time, and use software to complete translation tasks;

(2) Work efficiency: speed up the translation and carry out more translation in a short time;

(3) Memorability: simple operation and easy to use memory. For users who do not use it frequently, they can still find it easy to use after a period of time.

(4) Error rate: CAT software translation can help to maintain a low error rate and ensure the quality of translation.

(5) Subjective satisfaction: user experience is the most critical element of the software. Better communicative interface and more satisfactory translation results are the service tenet of the auxiliary translation software. 


\section{User experience}

User experience is the user's subjective feelings in the process of human-computer interaction between software and users. User experience is the key to user loyalty and software life cycle (Van Schaik, \& Ling, 2008).

The elements of user experience can be divided into different layers from the abstract one to specific one including strategy, scope, structure, frame and presentation. The strategy focuses on the user needs and business goals; scope focuses on the combination of current and inactive functions. The focuses of the software design are the software interaction design and information architecture; framework refers to the interface design and information design; as for presentation, visual design equips the software with better man-machine interaction interface (Bruseberg \& Mcdonagh-Philp, 2001; De Loor, Richard, \&, Bevacqua, 2017; Dhouib, Trabelsi, Kolski, \& Neji, 2017).

Each layer of the user experience is a progressive relationship, in which the upper layer determines the next layer while each layer has no intersection in space. The change of demand in the higher level will lead to the re-evaluation of the next layer. Therefore, the most important part of the user experience is the user needs and business needs, that is, the strategic layer (Peres, Meira, \& Luciano, 2003).

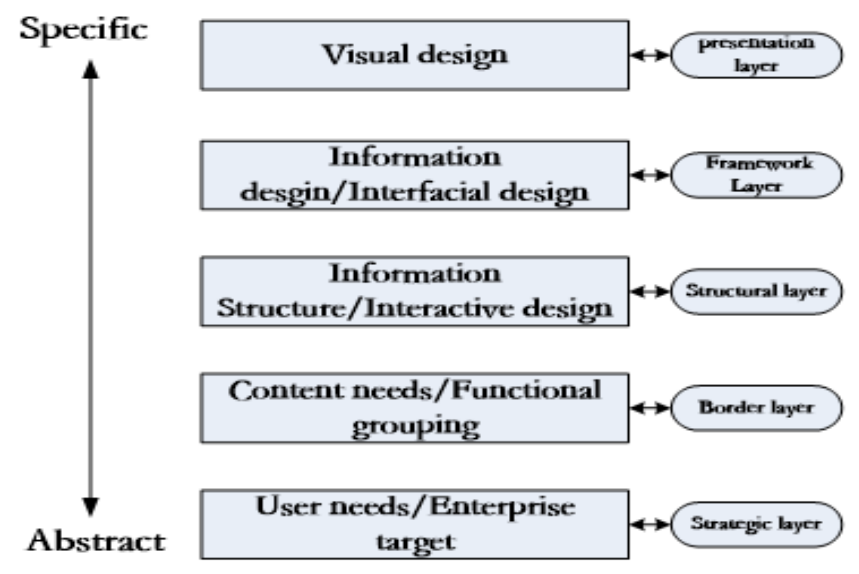

Figure 2. User experience element model

\section{Application evaluation of cat}

\section{Questionnaire survey and result statistics}

In this study, 150 questionnaires were collected from online questionnaires, and 115 valid questionnaires were remained after incomplete questionnaires were excluded. The questionnaire mainly consists of two parts: part one includes the form of translation, the year of experience, income of the users and their willing to accept CAT training. Part two studies the satisfaction of the respondents and their requirements on performance (Fiebach, \& Galinovic, 2011). 
Respondents mainly include professional translators and non-professional translators, in which nonprofessional translators include part-time interpreters, students major in translation or MTI and other translators who work in the translation industry.

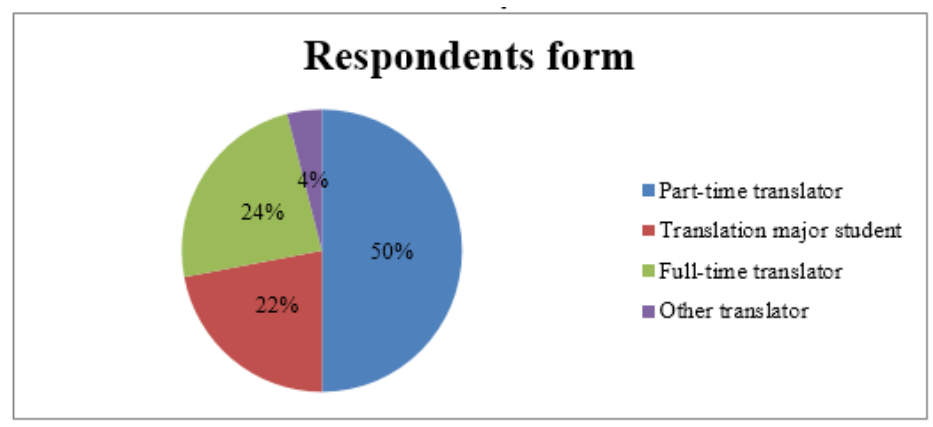

Figure 3. The form of the respondents

As seen in Figure 2, there are only $24 \%$ professional translators engaged in translation, and most of them are part-time translators. Although most of their translation experience is no more than three years, their average daily translation vocabulary has reached around 3000 words. The certain translation intensity makes the questionnaire a more valuable reference (Yun, 2006).

Table 1

Occupation and CAT Software Usage Tables

\begin{tabular}{|c|c|c|c|c|c|c|c|}
\hline Occupation & & & Occupation 1 & Occupation 2 & Occupation 3 & Occupation 4 & Total \\
\hline \multirow{6}{*}{ CAT } & \multirow{2}{*}{ CAT1 } & Count & 3 & 3 & 2 & 1 & 9 \\
\hline & & $\%$ of Total & $2.6 \%$ & $2.6 \%$ & $1.7 \%$ & $0.9 \%$ & $7.8 \%$ \\
\hline & \multirow{2}{*}{ CAT2 } & Count & 11 & 35 & 20 & 2 & 68 \\
\hline & & $\%$ of Total & $9.5 \%$ & $30.4 \%$ & $17.4 \%$ & $1.7 \%$ & $59.1 \%$ \\
\hline & \multirow{2}{*}{ CAT3 } & Count & 14 & 19 & 3 & 2 & 38 \\
\hline & & $\%$ of Total & $12.1 \%$ & $16.5 \%$ & $2.6 \%$ & $1.7 \%$ & $33.0 \%$ \\
\hline \multirow{2}{*}{ Total } & & Count & 28 & 57 & 25 & 5 & 115 \\
\hline & & $\%$ of Total & $24.3 \%$ & $49.6 \%$ & $21.7 \%$ & $4.3 \%$ & $100 \%$ \\
\hline
\end{tabular}

In table, career 1 is professional translator, career 2 is part-time translator, career 3 is students major in translation, and 4 refers to others; CAT1 is almost unused, CAT2 is occasionally used, CAT3 is frequently used. From the table, it can be seen that only $7.8 \%$ of the respondents did not have access to CAT software, indicating that people engaged in translation jobs use CAT software more frequently. Among them, translators who frequently use CAT software as auxiliaries account for $33 \%$.

In addition, according to the questionnaire, more than $90 \%$ of respondents received CAT software training, of which $37.4 \%$ received relatively professional trainings. More than $84.2 \%$ of respondents agree with the efficiency of CAT software, and think that CAT software has a significant effect on the improvement of work efficiency. No user thinks that CAT will reduce translation efficiency (Cettolo et al., 2014).

The investigation was carried out in order to study the respondents' subjective satisfaction. 7-point means very satisfied, 6 refers to satisfied, 4 refers to general, 3 is not satisfied, and 2 means not satisfied. The statistical results are shown in table 2. 
According to the statistical results, the average score of the subjective satisfaction of CAT software was 6 , indicating that respondents had relatively higher satisfaction with CAT software under the 1 7 score system.

Table 2

The Subjective Satisfaction Statistics

\begin{tabular}{lccccc}
\hline Subjective attitude & Very satisfied & Satisfied & General & Unsatisfied & Very unsatisfied \\
\hline Score & 7 & 6 & 4 & 3 & 1 \\
Count & 58 & 32 & 15 & 7 & 3 \\
\hline
\end{tabular}

\section{The impact of usability indexes on the choice of the users}

Research hypothesis. CAT software, like other computer software, needs to rely on the user's satisfaction to maintain the lifecycle of the software. The higher the user's satisfaction is, the more likely to continue to use the software. At the same time, the efficiency and the difficulty to use also affect the user's choice of CAT software. This paper presents the hypothesis: with high satisfaction, low error rate, short learning period and high efficiency, the users are more likely to use the CAT software (Civera et al., 2004).

Research design. For each item in the hypothesis, the user's satisfaction is obtained by 1-7 scoring software. Learnability (shorter learning period) refers to the time spent by the user from initial contact with the CAT software to use the software to deal with the translation project on a monthly basis; the error rate is calculated based on the number of major errors in the translation project which has been translated for 10 times, the unit of which is the number of times; translation efficiency is a categorical variable which is classified into 5 categories, namely, significantly improve, improve but not obvious, uncertain, decrease but not obvious, and significantly decrease (Koehn, 2009).

Model construction and assumption. According to the above hypothesis and the definitions of the variables, the regression equation of CAT software usage and each index is established (1):

$$
P=\frac{1}{1+\exp \left[-\left(\beta_{0}+\beta_{1} S+\beta_{2} W+\beta_{3} T+\beta_{4} E\right)\right]}
$$

Since efficiency is a classified variable, the regression equation is shown as follows:

$$
P=\frac{1}{1+\exp \left[-\left(\beta_{0}+\beta_{1} S+\beta_{2} W+\beta_{3} T+\beta_{4} E_{1}+\beta_{4} E_{2}\right)\right]}
$$

Among them, S represents satisfaction, W represents the times of the error, T represents learning cycle, E represents efficiency. The test of the variables in the regression equation is shown in Table 3.

According to the statistical results, except for the efficiency variable (0.143), the other variables are significant at the level of $10 \%$, among which the error rate and satisfaction were significant at the level of $5 \%$. The impact of indexes on the usability of CAT can be listed in descending order: satisfaction, error rate, learning cycle, work efficiency. Every one step the satisfaction improves, the advantage of CAT will increase by 3.18 times; if doubling the error rate and the learning cycle, the advantage of CAT will be increased by 0.45 times and 0.87 times separately. With the increase of error rate and the learning cycle, the usability of CAT software 
tends to decrease. Although the efficiency of the independent variable does not have statistical significance at $10 \%$ test level, the value of $\mathrm{P}$ is very close to 0.1 .

Table 3

Variables in the equation

\begin{tabular}{lcccccccc}
\hline Variables & B & S.E. & Wald & df & Sig. & Exp(B) & $\begin{array}{c}\text { Lower (95\% C.I. } \\
\text { for EXP(B)) }\end{array}$ & $\begin{array}{c}\text { Upper (95\% C.I. } \\
\text { for EXP(B)) }\end{array}$ \\
\hline Efficiency & & & 3.885 & 2 & 0.143 & & & \\
\hline Efficiency (1) & 2.792 & 1.699 & 2.701 & 2 & 0.100 & 16.315 & 0.584 & 455.566 \\
\hline Efficiency (2) & 2.141 & 1.419 & 2.275 & 1 & 0.131 & 8.508 & 0.527 & 137.407 \\
\hline Error rate & $\begin{array}{c}-0801 \\
0\end{array}$ & 0.378 & 4.496 & 1 & 0.034 & 0.449 & 0.214 & 0.941 \\
\hline $\begin{array}{l}\text { Learning } \\
\text { cycle }\end{array}$ & 0.136 & 0.080 & 2.900 & 1 & 0.089 & 0.873 & 0.746 & 1.021 \\
\hline Satisfaction & 1.157 & 0.455 & 6.47 & 1 & 0.779 & 3.180 & 1.304 & 7.756 \\
\hline Constant & -.608 & 2.164 & 0.079 & 1 & 0.100 & 0.544 & - & - \\
\hline
\end{tabular}

Therefore, the hypothesis in this paper is verified, that is, higher satisfaction, lower error rate, shorter learning cycle and higher efficiency will make users more inclined to use CAT software.

\section{The application of cat in college English translation teaching}

Since the Ministry of Education attaches much weight translation and translation major have been recognized by the society, computer assisted translation teaching has made great progress in Chinese colleges and universities. At present, the country has more than 200 MTI pilots. The application of CAT in college English translation teaching was also investigated by questionnaires, in which teachers and students' subjective recognition of CAT was collected. Interviews were used to understand the current teaching strategies of CAT (Huang, Yang, Yang \& Chang, 2013).

\section{Teachers' cognition of CAT teaching}

Questionnaire is used in studying the teachers' attitudes on the domestic CAT software teaching. For 39 College English teachers in various colleges and universities in China, this paper puts forward a questionnaire with the question of "whether you think the current CAT software teaching is successful or not". The questionnaire was distributed through network, and 39 questionnaires were recollected.

Table 4

The Teacher's Survey Results

\begin{tabular}{lcc}
\hline Option & subtotal & proportion \\
\hline Very successful, and achieved very good results & 3 & $7.7 \%$ \\
\hline No different from other educational software & 7 & $17.9 \%$ \\
\hline There are many difficulties & 16 & $41 \%$ \\
\hline Do not understand, cannot answer & 13 & $33.3 \%$ \\
\hline
\end{tabular}

The table reflects the actual situation of CAT teaching in college is not optimistic. Only $7.7 \%$ of the teachers thought that CAT had achieved good results in translation teaching. More than $33.3 \%$ teachers did not know much about CAT, which not only reflects that the majority of teachers do not pay much attention to CAT teaching, but also indicates CAT is not highly accepted by college students in China. 
Wu, Zhang / Evaluation of Computer Assisted Translation and its Teaching Application in English Translation

The majority of English teachers should learn CAT technology from their own point of view, and master the usability evaluation index of CAT. They should help students improve the ability of English translation by setting reasonable CAT teaching cases, and at the meantime, promote the application of CAT in teaching.

\section{Students' cognition of CAT}

Students' knowledge of computer aided translation was investigated by distributing 65 web questionnaires. The question is "what attitude do you take towards CAT software?"". The results are shown in table 5.

Table 5

The Student's Survey Results

\begin{tabular}{lcc}
\hline Option & subtotal & proportion \\
\hline Completely sure & 8 & $12.3 \%$ \\
\hline Tendency sure & 46 & $70.8 \%$ \\
\hline Cannot tell & 8 & $13.8 \%$ \\
\hline Tendency deny & 2 & $3.1 \%$ \\
\hline Completely deny & 0 & $0 \%$ \\
\hline
\end{tabular}

It can be seen from the table that most of the students' attitudes towards CAT software are completely affirmative or tend to be affirmative, accounting for $83.1 \%$ of the total. It reflects the students' acceptance of the teaching mode of CAT, which contrasts sharply with the teaching attitudes of teachers. It further reflects the contradiction of the supply and demand of CAT teaching in College English translation education. It is necessary to further promote the application of CAT technology in translation teaching.

\section{Conclusion}

With the development of computer technology, CAT has more and more functions, and the popularization continues to expand. In this paper, the application of CAT software is evaluated based on the user requirements and the usability evaluation indexes. First, the relative concepts of CAT technology are defined, and then questionnaire survey is carried out to analyze the evaluation on the application of CAT. Finally, its application in college English translation teaching is analyzed. The main conclusions are as follows:

(1) This paper evaluates CAT software from the user's point of view, which is conducive to the mutual promotion and coordinated development of the CAT software and the user's choice.

(2) High satisfaction, low error rate, short learning recycle and high efficiency will make users tend to use the CAT software.

(3) There is a contradiction between the supply and demand in the application of CAT in universities. CAT software should be more widely used in teaching. 


\section{References}

Barrachina, S., Bender, O., Casacuberta, F., Civera, J., Cubel, E., Khadivi, S., Lagarda, A., Ney, H., Tomas, J., Vidal, E. \& Vilar, J.M. (2009). Statistical approaches to computer-assisted translation. Computational Linguistics, 35(1), 3-28. http://dx.doi.org/10.1162/coli.2008.07-055-R2-06-29

Bruseberg, A. \& Mcdonagh-Philp, D. (2001). New product development by eliciting user experience and aspirations. International Journal of Human-Computer Studies, 55(4), 435-452. http://dx.doi.org/10.1006/ijhc.2001.0479

Cettolo, M., Bertoldi, N., Federico, M., Schwenk, H., Barrault, L. \& Servan, C. (2014). Translation project adaptation for MT-enhanced computer assisted translation. Machine Translation, 28(2), 127-150. http://dx.doi.org/10.1007/s10590-014-9152-1

Civera, J., Vilar, J.M., Cubel, E., Lagarda A.L., Barrachina S., Casacuberta F., Vidal, E., Picó, D. \& González, J. (2004). A syntactic pattern recognition approach to computer assisted translation. Lecture Notes in Computer Science, 3138, 207-215. http://dx.doi.org/10.1007/978-3-540-27868-9_21

De Loor, P., Richard, R. \& Bevacqua, E. (2017). Evolutive body interaction between a human and a virtual character. Theoretical model proposition and evaluation within a fitness exergame. Revue d'Intelligence Artificielle, 31(5), 557-579. http://dx.doi.org/10.3166/RIA.31.557-579

Dhouib, A., Trabelsi, A., Kolski, C., \& Neji, M. (2017). Prioritizing the usability criteria of adaptive user interfaces of information systems based on ISO/IEC 25040 standard, Ingenierie des Systemes d'Information, 22(4), 107-128. http://dx.doi.org/10.3166/ISI.22.4.107-128

Fiebach, J.B. \& Galinovic, I. (2011). Explanation of our methodological approach to perfusion software evaluation: Reply to the letter by Chaibi and Boutelier. Cerebrovascular Diseases, 32(5), 515-515. http://dx.doi.org/10.1159/000329312

Huang, C.C., Yang, P.C., Yang, P.C. \& Chang, J.S. (2013). A computer-assisted translation and writing system. ACM Transactions on Asian Language Information Processing, 12(4), 15-15. http://dx.doi.org/10.1145/2505984

Hutchins, W.J. \& Somers, H.L. (1992). An introduction to machine translation. Journal of Solution Chemistry, 33(3), 1059-1066.

Koehn, P. (2009). A process study of computer-aided translation. Machine Translation, 23(4), 241-263. http://dx.doi.org/10.1007/s10590-010-9076-3

Mileto, F. (2014). From computer assisted translation to human assisted translation. Annals of Emergency Medicine, 40(1), e83-e101.

Miquel, E.G., Felipe, S.M. \& Mikel, L.F. (2015). Using machine translation to provide target-language edit hints in computer aided translation based on translation memories. Journal of Artificial Intelligence Research, 53, 169-222.

Peres, F. \& Meira, L. (2003). Educational software evaluation centered on dialogue: Interface, collaboration and scientific concepts. Human Brain Mapping, 32(1), 80-80. http://dx.doi.org/10.1145/944519.944530

Vakil, Z. \& Khadivi, S. (2013). Development and enhancement of an interactive computer-assisted translation system for English to Persian (written in Persian language). Journal of Hydraulic Engineering, 138(10), 
Wu, Zhang / Evaluation of Computer Assisted Translation and its Teaching Application in English Translation

897-901.

Van Schaik, P. \& Ling, J. (2008). Modelling user experience with web sites: usability, hedonic value, beauty and goodness. Interacting with Computers, 20(3), 419-432. http://dx.doi.org/10.1016/j.intcom.2008.03.001

Yun, W.U. (2006). The application of computer-aided translation systems in translation teaching. ComputerAssisted Foreign Language Education.

Zhang, E. \& Zhang, W. (2013). Application of computer-aided translation technology in translation teaching. International Journal of Emerging Technologies in Learning, 8(5), 15. http://dx.doi.org/10.4156/aiss.vol5.issue5.22 